\title{
ON THE LOCAL FORM OF THE SECOND LAW OF THERMODYNAMICS IN CONTINUUM MECHANICS*
}

\author{
BY \\ L. C. WOODS \\ Mathematical Institute, Oxford University
}

Summary. The Clausius-Duhem inequality $\sigma(\mathbf{r}, t) \geq 0$, a widely adopted axiom in continuum mechanics, leads to the conclusion that for many materials the entropy $s$ cannot depend on gradients like the temperature gradient $\mathbf{g}$ and the velocity gradient $\mathbf{e}$. But this is at variance with the received view (since Gibbs) that entropy is a function of thermodynamic state, however detailed that state description may be. Gradients, and even higher derivatives of macroscopic variables, may be included as state variables (although only on macroscopic time scales shorter than or comparable with their natural relaxation times), and the fundamental property of entropy is its convexity - the more detailed the specification of state, the smaller is the corresponding value of entropy.

The entropy of a perfect monatomic gas is evaluated via the maximum principle, on the assumption that $\mathbf{g}$ and $e$ are state coordinates, and it is found that $s$ does depend on $\mathbf{g} \cdot \mathbf{g}$ and $\mathbf{e}: \mathbf{e}$, contrary to statements appearing in many textbooks on continuum mechanics. The source of the error in these works is shown to lie in applying $\sigma \geq 0$ to relations involving second derivatives. The correct form of the Clausius-Duhem inequality contains only first-order derivatives; that is, it must be confined to linear constitutive relations. In this form the inequality is a consequence of the convexity of $s$, which is a somewhat more general manifestation of the second law.

1. Introduction. There is a wide-spread but erroneous belief held by many authors of papers and books in continuum mechanics ${ }^{1}$ that entropy is a function of state, which like any other state function, has a precise value in any prescribed thermo-mechanical phenomenon. Clearly functions like the internal energy per unit mass $u$, the density $\rho$, and the material velocity $\mathbf{v}$ can be assigned precise values at a point $P(\mathbf{r}, t)$ of a continuum, for these quantities have physical meanings independent of the extent of the observer's knowledge of "conditions" at $P$. These conditions may include space and time derivatives of first, second and even higher order of macroscopic variables, and the difficulty is that while $u, \rho, \mathbf{v}, \ldots$ can be assigned independently of such derivatives, the specific entropy $s(\mathbf{r}, t)$ cannot-or at least not without making approximations. The entropies we use in macroscopic physics are always approximating functions, and the same must be true of derived functions like the free energy,

$$
\psi=u-T s
$$

\footnotetext{
* Received April 3, 1980.

${ }^{1}$ E.g., see Eringen (1975), Jaunzemis (1967), Truesdell and Noll (1965) and Leigh (1968).
} 
where $T=T(\mathbf{r}, t)$ is the temperature. (To avoid tainting temperature with the same uncertainty that is unavoidably inherent in $s$, we must define it via a state equation containing only "precise" variables like $u$ and $\rho$.)

The rate of production of entropy per unit volume at $P(\mathbf{r}, t)$, say $\sigma(\mathbf{r}, t)$, is related to $s$ and its flux $\mathbf{J}_{s}$ by

$$
\sigma=\rho D s+\nabla \cdot \mathbf{J}_{s}-Q / T \quad\left(D \equiv \frac{\partial}{\partial t}+\mathbf{v} \cdot \nabla\right),
$$

where $D$ is the material derivative and $Q$ is the heat supplied per unit volume by radiation. It is usually assumed that

$$
\mathbf{J}_{s}=\mathbf{q} / T,
$$

where $\mathbf{q}$ is the heat flux vector. Accepting that at best $s$ is an approximating function, we infer that $\sigma$ and $\mathbf{J}_{s}$ in (2) must also lack precision; in fact, it can be shown that (3) is correct only in a theory confined to first derivatives at $P$ (Woods, 1980).

The second law of thermodynamics requires that the integral of $\sigma$ over the volume $V$ of the thermomechanical system be non-negative for an appreciable time interval, i.e.

$$
\int_{t_{1}}^{t_{2}} \int_{V} \sigma(\mathbf{r}, d t) d \mathbf{r} d t \geq 0 .
$$

The local form of this inequality, often termed the Clausius-Duhem inequality,

$$
\sigma(\mathbf{r}, t) \geq 0,
$$

follows from the assumption that (4) holds for all parts $P$ of $V$ and for all time intervals $t_{2}-t_{1}$ however small $P$ and $t_{2}-t_{1}$ may be. For constitutive relations involving firstorder derivatives only, it has been widely and successfully employed (e.g., see de Groot and Mazur (1962), Haase (1968), or Woods (1975)). In this case the entropy is defined via a Clausius-Gibbs relation

$$
T d s_{0}=d u+p d \rho^{-1}+\cdots
$$

in which $s_{0}=s(u, \rho, \ldots)$ depends only on the equilibrium state at $P$, i.e. does not depend on any derivatives at $P$.

Unfortunately, most writers in continuum mechanics, apparently unaware of the approximating nature of the functions $s, \mathbf{J}_{s}$ and $\sigma$, have pressed the theory beyond first-order derivatives, accepting the inequality in (5) as being true without limit on the extent of the observer's knowledge of conditions at $P$. The law in (4) is statistical, but a large volume $V$ and a substantial time interval $t_{2}-t_{1}$ suppress local fluctuations, so its violation is extremely unlikely. But (5) is in a much weaker position to withstand fluctuations; the more detailed the knowledge assumed about conditions at $P$, the more likely it is that the inequality can be violated via the higher-order derivatives in singular regions where the first derivatives are negligible.

In Sec. 2 we shall give an example of the misuse of the local inequality (5), employing it to argue that $s$ must be independent of the temperature gradient. But this is in contradiction to the fact that there can be no constraints on the dependency of $s$, other than that it satisfy the entropy maximum principle. In fact, entropy is like a bottomless pit so far as its functional dependence on derivatives of higher and higher order is concerned. Fortunately, the order of the expansion parameter $\varepsilon$ in this development of $s$ 
is the same as the order of the derivatives, so that approximations in which terms $O\left(\varepsilon^{n}\right)$ can be ignored also eliminate derivatives of order $n$ and higher. In Sec. 3 we shall illustrate this point by obtaining the formula giving $s$ as a function of temperature and velocity gradients in a monatomic perfect gas. Our final conclusion is that the local form of the inequality holds only for constitutive relations linear in first derivatives, and that the convexity of $s$ as a function of state should replace the inequality in theories concerned with nonlinear constitutive relations.

\section{1. "Proof" that entropy is independent of temperature gradient. Let}

$$
\mathbf{e} \equiv \nabla \mathbf{v}, \quad \mathbf{g}=\nabla T
$$

be the gradients of interest in what follows. Energy balance at $P$ gives

$$
\rho D u+\mathbf{p}: \mathbf{e}+\nabla \cdot \mathbf{q}-Q=0,
$$

where $-p$ is the stress tensor. Hence by (1)-(5)

$$
T \sigma=-\rho D \psi-s \rho D T-\mathbf{p}: \mathbf{e}-\mathbf{g} \cdot \mathbf{q} / T \geq 0 .
$$

We shall apply this inequality to the case of a perfect monatomic gas in order to relate the outcome to the kinetic theory to be presented in Sec. 3. What follows is a simple extension of the argument applied to a "thermoelastic" fluid appearing on pp. 190-192 of Leigh's (1968) text on nonlinear continuum mechanics, and in many other places. ${ }^{2}$ The more general case of a simple material with fading memory was treated by Coleman (1963). The conclusion that $s$ does not depend on $\mathbf{g}$ is quite general-and quite wrong.

Following a pattern familiar in rational mechanics, we choose $\rho, T, \mathbf{g}$ and $\mathbf{e}$ to be independent variables, and postulate that each of $s, \psi, \mathbf{p}$ and $\mathbf{q}$ depend on this set, i.e.

$$
\begin{aligned}
s & =s(\rho, T, \mathbf{g}, \mathbf{e}), & \psi & =\psi(\rho, T, \mathbf{g}, \mathbf{e}), \\
\mathbf{p} & =\mathbf{p}(\rho, T, \mathbf{g}, \mathbf{e}), & \mathbf{q} & =\mathbf{q}(\rho, T, \mathbf{g}, \mathbf{e}) .
\end{aligned}
$$

By continuity and the definition

$$
\boldsymbol{\pi} \equiv \mathbf{p}-\mathbf{p} \mathbf{1}
$$

where $p$ is the pressure and 1 the unit tensor, we can write

$$
\mathbf{p}: \mathbf{e}=p \nabla \cdot \mathbf{v}+\boldsymbol{\pi}: \mathbf{e}=-\frac{p}{\rho} D \rho+\boldsymbol{\pi}: \mathbf{e} .
$$

This result and (8) enable us to write (7) in the form

$$
-\boldsymbol{\pi}: \mathbf{e}-\mathbf{q} \cdot \mathbf{g} / T-\rho\left(\frac{\partial \psi}{\partial \rho}-\frac{p}{\rho^{2}}\right) D \rho-\rho\left(\frac{\partial \psi}{\partial T}+s\right) D T-\frac{\partial \psi}{\partial \mathbf{g}} \cdot D \mathbf{g}-\frac{\partial \psi}{\partial \mathbf{e}}: D \mathbf{e} \geq 0 .
$$

Since the balance equations may be used to define body forces and thermal sources, any choice of $D \rho, D T, D \mathbf{g}, D \mathbf{e}$ is possible. Therefore (10) requires that

$$
T \sigma=-\boldsymbol{\pi}: \mathbf{e}-\mathbf{q} \cdot \mathbf{g} / T \geq 0,
$$

\footnotetext{
${ }^{2}$ For a recent example, see Eq. (3.46) of Fosdick and Rajagopal (1980).
} 
and

$$
\frac{\partial \psi}{\partial \rho}=\frac{p}{\rho^{2}}, \quad \frac{\partial \psi}{\partial T}=-s, \quad \frac{\partial \psi}{\partial \mathbf{g}}=0, \quad \frac{\partial \psi}{\partial \mathbf{e}}=0 .
$$

We conclude that $\psi$ and $s$ depend only on $\rho$ and $T$; in fact, (1) and (12) yield the Clausius-Gibbs relation (6).

The main conclusion we wish to note here is that $s$ is independent of the gradients $\mathbf{g}$ and e. Were this result viewed as being approximate only, and the inequality in (11) therefore correct only to the same order of approximation, there would be no conflict with the remarks made in the final paragraph of Sec. 1, nor with the analysis to follow. But the above approach does not lend itself to this interpretation, as no small parameter is evident in the analysis.

3. The dependence of the entropy of a perfect gas on macroscopic gradients. Let $f(\mathbf{r}, \mathbf{w}, \mathbf{t}) d \mathbf{w} d \mathbf{r}$ be the number of molecules lying in the volume element $d \mathbf{w} d \mathbf{r}$ of phase space; then the fluid velocity at $P(\mathbf{r}, t)$ is given by

$$
\rho \mathbf{v}=\int m \mathbf{w} f d \mathbf{w},
$$

where the integral here (and those to follow) is understood to extend over the whole of velocity space, and $m$ is the molecular mass: also the mass density and energy density are

$$
\rho=m n=\int m f d \mathbf{w}, \quad \rho u=\int \frac{1}{2} m c^{2} f d \mathbf{w},
$$

where $n$ is the number density and $\mathbf{c}=\mathbf{w}-\mathbf{v}$ is the random component of molecular velocity. Shortly we shall also need the entropy production rate per unit volume (Woods, 1975, p. 315)

where

$$
\sigma=-k \int \partial f \ln \left(f / f_{0}\right) d \mathbf{w}=-k \int\left(\frac{\partial f}{\partial t}\right)_{\mathrm{col}} \ln \left(\frac{f}{f_{0}}\right) d \mathbf{w}
$$

$$
\mathscr{D} \equiv \frac{\partial}{\partial t}+\mathbf{w} \cdot \frac{\partial}{\partial \mathbf{r}}+\dot{\mathbf{w}} \cdot \frac{\partial}{\partial \mathbf{w}}=D+\mathbf{c} \cdot \nabla+\mathbf{F} \cdot \frac{\partial}{\partial \mathbf{w}},(\mathbf{F}=\dot{\mathbf{w}})
$$

is the time rate of change following a bunch of molecules through phase space, $k$ is Boltzmann's constant, $f_{0}$ is the equilibrium distribution defined below, $\mathbf{F}$ is the body force per unit mass and $(\partial f / \partial t)_{\mathrm{col}}$ is the rate of change of $f$ due to molecular collisions, which by Boltzmann's equation is equal to $2 \mathrm{ff}$.

The kinetic entropy is defined to within a constant by

$$
\rho s=-k \int f(\ln f-1) d \mathbf{w},
$$

and the maximum principle requires this to be maximized subject to any constraints on $f$ known to exist. As is well known, when this is done with prescribed values of $\rho, \mathbf{v}$ and $u$, i.e. with the constraints on $f$ given in (13) and (14), the outcome is Maxwell's equilbrium distribution

$$
f_{0}=n\left(\frac{m}{2 \pi k T}\right)^{3 / 2} e^{-m c^{2} / 2 k T},
$$


and the corresponding entropy is

$$
\rho s_{0}=-k \int f_{0}\left(\ln f_{0}-1\right) d \mathbf{w},
$$

which agrees with the value defined in (6).

Now suppose that, if in addition to given values for $\rho, \mathbf{v}$ and $u$, we have a given value for the rate $\sigma$ defined in $(15)_{1}$, or at least that part of $\sigma$ that depends on the gradients $\mathbf{g}$ and e (cf. 11)). Maximizing $\rho$ s subject to the constraints in (13), (14) and (15), we find

$$
\ln f+\alpha+\gamma \cdot \mathbf{c}+\beta c^{2}+\tau \mathscr{D} \ln f=0,
$$

where $\alpha, \gamma, \beta$ and $\tau$ are Langrangian multipliers. In equilibrium $\tau=0$, so to solve (20) by iteration we set $\tau \mathscr{D}=O(\varepsilon)$, where $\varepsilon$ is small, and expand $f$ in the form

$$
f=e^{\phi_{0}+\phi_{1}+\phi_{2}+\cdots} \quad\left(\phi_{n}=O\left(\varepsilon^{n}\right)\right) .
$$

Applying the constraint on entropy production rate to highest order only, we find from (20) and (21) that

$$
f=f_{0}\left\{1+\phi_{1}+\left(\phi_{2}+\frac{1}{2} \phi_{1}^{2}\right)+\cdots\right\}, \quad \phi_{1}=-\tau \mathscr{D} \ln f_{0},
$$

provided

$$
\int f_{0}\left[\begin{array}{l}
1 \\
\mathbf{c} \\
c^{2}
\end{array}\right]\left[\phi_{1}, \quad\left(\phi_{2}+\frac{1}{2} \phi_{1}^{2}\right), \quad\left(\phi_{3}+\phi_{2} \phi_{1}+\frac{1}{6} \phi_{1}^{3}\right), \ldots\right] d \mathbf{w}=0,
$$

in order that the constraints in (14) be satisfied to all orders. This can be ensured by writing $\alpha=\alpha_{0}+\alpha_{1}+\cdots, \gamma=\gamma_{0}+\gamma_{1}+\cdots, \beta=\beta_{0}+\beta_{1}+\cdots$, and using (23) to define the values of $\alpha_{r}, \gamma_{r}, \beta_{r}, r=1,2, \ldots$ (cf. Sec. 7.31 of Chapman and Cowling, 1970).

From (15) and (22),

$$
\sigma=\sigma_{1}+\sigma_{2}+\cdots
$$

where

$$
\begin{aligned}
\sigma_{1} & =\frac{k}{\tau} \int f_{0} \phi_{1}^{2} d w, \\
\sigma_{2} & =\frac{k}{\tau} \int f_{0}\left(\phi_{1} \phi_{2}+\phi_{1}^{3}-\frac{1}{2} \tau \mathscr{D} \phi_{1}^{2}\right) d w,
\end{aligned}
$$

If $\sigma_{1}$ is known, then $(22)_{2}$ and $(24)_{2}$ yield a value for $\tau$, although the physical significance of $\tau$ is to be found in $(\partial f / \partial t)_{\mathrm{col}}$, and is in fact proportional to the mean-free-time between successive molecular collisions.

To calculate $\phi_{1}$ from $(22)_{2}$ we use (18), plus the adiabatic relations $p \propto T^{5 / 2}$, $\rho \propto T^{3 / 2}$, which, being correct to $O(\varepsilon)$, are sufficient at this stage. (See discussion in 
chapter 7 of Chapman and Cowling, 1970.) We also use the equations of continuity and motion. Hence

$$
\mathscr{l} \ln f_{0}=-\frac{m}{2 k} \mathscr{L}\left(\frac{c^{2}}{T}\right)=-\frac{m}{2 k T}\left(\frac{1}{3} c^{2} \nabla \cdot \mathbf{v}+\mathbf{c} \cdot \nabla T-\mathbf{c} \cdot \mathscr{L}\right)
$$

where

$$
\mathscr{C} \mathbf{c}=\mathscr{W} \mathbf{w}-D \mathbf{v}-\mathbf{c} \cdot \nabla \mathbf{v}=\mathbf{F}-D \mathbf{v}-\mathbf{c} \cdot \nabla \mathbf{v}=\frac{5}{2} \frac{k}{m} \nabla T-\mathbf{c} \cdot \nabla \mathbf{v},
$$

so that

$$
\phi_{1}=\tau\left(\frac{5}{2}-\frac{m c^{2}}{2 k T}\right) \mathbf{c} \cdot \nabla \ln T-\tau \frac{m}{k T}\left(\mathbf{c c}-\frac{1}{3} c^{2} \mathbf{1}\right): \nabla \mathbf{v}
$$

This result can be generalized by noting that the dissipations due to gradients $\mathbf{g}=\nabla T$ and $\mathbf{e}=\nabla \mathbf{v}$ proceed on slightly different time scales. Thus $\sigma_{1}(\mathbf{g}, \mathbf{e})$, the leading term in (15), can be split into two independent components, $\sigma_{11}=\sigma_{1}(0$, e) and $\sigma_{12}=\sigma_{1}(\mathrm{~g}, 0)$, and we maximize $\rho s$ subject to each component being held constant. And if further we hold the integrands of $\sigma_{11}$ and $\sigma_{12}$ constant, the associated Lagrangian multipliers may be functions of $c$. This modification replaces (25) by

$$
\phi_{1}=\tau_{2}(c)\left(\frac{5}{2}-\frac{m c^{2}}{2 k T}\right) \mathbf{c} \cdot \nabla \ln T-\tau_{1}(c) \frac{m}{k T}\left(\mathbf{c c}-\frac{1}{3} c^{21}\right): \nabla \mathbf{v}
$$

$\tau_{2}(c), \tau_{1}(c)$ being the relaxation times for energy and momentum transport respectively. Then $(24)_{2}$ is replaced by

$$
\sigma_{1}=k \int f_{0} \tau_{1}^{-1} \phi_{11}^{2} d \mathbf{w}+k \int f_{0} \tau_{2}^{-1} \phi_{12}^{2} d \mathbf{w}
$$

where $\phi_{12}$ is the first term on the right of (26) and $\phi_{11}$ is the second. For the purposes of this paper, there is no real loss in taking $\tau_{1}$ and $\tau_{2}$ to be constants and related by $\tau_{2}=\frac{3}{2} \tau_{1}$; this is the case of Maxwellian molecules, which we shall adopt below.

Then with the definitions

$$
\mu=p \tau_{1}, \quad k=\frac{5}{2} \frac{k}{m} p \tau_{2}=\frac{5}{2} c_{v} \mu
$$

and the linear constitutive relations

$$
\mathbf{q}_{1}=-k \nabla T, \quad \boldsymbol{\pi}_{1}=-2 \mu \stackrel{\circ}{\nabla \mathbf{v},}
$$

(26) and (27) yield

$$
T \sigma_{1}=-\boldsymbol{\pi}_{1}: \mathbf{e}-\mathbf{q}_{1} \cdot \mathbf{g} / T,
$$

which should be compared with (11). 
By (17), (19), (21) and (23) we find that

$$
\rho s=\rho s_{0}+\rho s_{2}+\cdots
$$

where

$$
\begin{aligned}
& \rho s_{2}=-\frac{1}{2} k \int f_{0} \phi_{1}^{2} d \mathbf{w}, \\
& \rho s_{3}=-k \int f_{0}\left(\phi_{2}+\frac{1}{3} \phi_{1}^{2}\right) \phi_{1} d \mathbf{w}, \\
& \rho s_{4}=-k \int f_{0}\left(\frac{1}{2} \phi_{2}^{2}+\frac{1}{8} \phi_{1}^{4}+\phi_{1} \phi_{3}+\phi_{1}^{2} \phi_{2}\right) d \mathbf{w},
\end{aligned}
$$

Thus by (26)

$$
\rho s=\rho s_{0}-\frac{p}{T}\left(\frac{5}{4} \tau_{2}^{2} \nabla T \cdot \nabla T+\tau_{1}^{2} \nabla^{0} \mathbf{v}: \nabla^{0} \mathbf{v}\right)+O\left(\varepsilon^{3}\right) .
$$

Notice from (27) and (31) that

$$
\rho s_{2}=-\frac{1}{2} \tau_{1} \sigma_{11}-\frac{1}{2} \tau_{2} \sigma_{12} \quad\left(\tau_{1}, \tau_{2} \geq 0\right),
$$

a result recently obtained (Woods, 1980) by a more general if less precise argument not restricted to the case of a perfect monatomic gas. The constraints on $\tau_{1}$ and $\tau_{2}$ follow from the convexity condition $\rho s \leq \rho s_{0}$. Only if terms $O\left(\varepsilon^{2}\right)$ can be ignored is it true that entropy is independent of $\nabla T$. Eq. (32) is not new; it was obtained by Shavit and Zvirin (1970) by a somewhat different route.

It is a reasonable inference from the above example that if in the constitutive relations for any medium, terms non-linear in the space derivatives of macroscopic variables are retained, then the entropy must be a convex function of these derivatives.

4. The restriction on the Clausius-Duhem relation. Our remaining task is to find the error in the theory presented in Sec. 2 which gave $s$ independent of $\nabla T$ in contradiction to the correct result given in Sec. 3, also of course in contradiction to the quite general fact that minus entropy is a measure of our knowledge of the "state" of a system, and "state" can depend on the derivatives of macroscopic variables of any order.

We start by observing from (24) that $\sigma_{1} \geq 0$, but that $\sigma_{2}$ may have either sign. In general $\sigma_{1} \gg\left|\sigma_{2}\right|$ because $\sigma_{2}$ is a factor $\varepsilon$ smaller than $\sigma_{1}$. However, if $\phi_{1}=0$ in a small, singular region, then in that region

$$
\sigma=\sigma_{2}=-\left.\frac{1}{2} k \int f_{0} \mathscr{D} \phi_{1}^{2}\right|_{\phi_{1}=0} ^{d w} d \mathbf{w},
$$

which is unrestricted in sign, i.e. the Clausius-Duhem inequality in its general form (5) does not hold. In fact, it is clear that the only valid form of this inequality we can take is

$$
\sigma_{1} \geq 0 \text {, }
$$

which means that (11) is correct only if $\sigma, \pi$ and $\mathbf{q}$ are replaced by $\sigma_{1}, \pi_{1}$ and $\mathbf{q}_{1}$ (see (29)). The terms involving the second-order derivatives $D g$ and $D e$ in (10) must be 
omitted and the conclusions in $(12)_{3}$ and $(12)_{4}$ do not follow. The author has also shown (35) to be the correct form of the Clausius-Duhem inequality by less precise arguments not involving kinetic theory (Woods, 1980).

From (31) we see that even when $\phi_{1}$ is zero and $\sigma$ unconstrained in sign, it still follows that $s<s_{0}$, for in this case

$$
\rho s=\rho s_{0}-\rho s_{4}-\cdots=\rho s_{0}-\frac{1}{2} k \int f_{0} \phi_{2}^{2} d \mathbf{w}-\cdots .
$$

This convexity of $s$ is an entirely reliable form of the second law of thermodynamics. Indeed, the inference that $\sigma_{1} \geq 0$ follows from convexity in the form $s_{2} \leq 0$ plus the relation between $\sigma_{1}$ and $s_{2}$ given in (33). And if one traces the origin of the ClausiusDuhem inequality (e.g. see Woods, 1975, Sec. 12.3) one finds that it is based on Clausius' inequality, which involves increases in entropy, but no time derivatives. These increases are a consequence of requiring our thermodynamic systems to be stable; they imply that the greater the constraints on the system the smaller the entropy, and this is equivalent to requiring entropy to be a convex function of its arguments. The introduction of (a) time derivatives and (b) infinitesimally small systems are each recipes for destroying the underlying inequality, even though averages over long times and large systems still obey the law of entropy increase. Fortunately (35) remains, but this is all.

It is curious that the convexity of entropy as a function of state is quite ignored in the works of Noll, Coleman, Truesdell, Eringen and the many others, who have been content to accept the Clausius-Duhem inequality (in its general, nonlinear form) as a suitable axiom for their exercises in rational continuum mechanics.

\section{REFERENCES}

[1] B. D. Coleman, Arch. Rat. Mech. Anal. 17, 1-46, 230-254 (1964)

[2] S. R. de Groot and P. Mazur, Non-equilibrium thermodynamics, North Holland Pub. Co. (1962)

[3] A. C. Eringen (ed.), Continuum physics, Vol. II, Academic Press, 115-126 (1975)

[4] R. L. Fosdick and K. R. Rajagopal, Proc. Roy. Soc. A369, 351-377 (1980)

[5] H. Grad, Comm. Pure Applied Math. 2, 331 (1949)

[6] R. Haase, Thermodynamics of irreversible processes, Addison-Wesley (1968)

[7] W. Jaunzemis, Continuum mechanics, Macmillan (1967)

[8] D. C. Leigh, Non-linear continuum mechanics, McGraw-Hill (1967)

[9] A. Shavit and Y. Zvarin, Technion Report TME-110, Haifa (1970)

[10] C. Truesdell and W. Noll, The non-linear field theories of mechanics, in Handbuch der Physik (ed. Flugge) III/3, Springer, Berlin (1965)

[11] L. C. Woods, The thermodynamics of fluid systems, Oxford (1975)

[12] L. C. Woods, J. Fluid Mech. 101, 225-241 (1980) 